Pesq. Vet. Bras. 35(11):899-905, novembro 2015 DOI: $10.1590 / \mathrm{S} 0100-736 \mathrm{X} 2015001100005$

\title{
Dioctophyma renale em 28 cães: aspectos clinicopatológicos e ultrassonográficos ${ }^{1}$
}

\author{
Caroline S. Silveira ${ }^{2}$, Aline Diefenbach ${ }^{3}$, Maria Lígia Mistieri ${ }^{4}$ Ingrid R.L. Machado ${ }^{5}$ \\ e Bruno L. Anjos ${ }^{6 *}$
}

\begin{abstract}
Silveira C.S., Diefenbach A., Mistieri M.L, Machado R.L. \& Anjos B.L. 2015. [Dioctophyma renale in 28 dogs: clinicpathological and ultrasonographic findings.] Dioctophyma renale em 28 cães: aspectos clinicopatológicos e ultrassonográficos. Pesquisa Veterinária Brasileira 35(11):899-905. Programa de Pós-Graduação em Ciência Animal, Universidade Federal do Pampa, Uruguaiana, RS 97500-970, Brazil. E-mail: anjosbl@gmail.com

Dioctophyma renale infection in dogs is being considered uncommon for most parts of the world. However, some regions show an increase of this infection; but many data of the epidemiology and the biological cycle of the parasite are still unclear. The present study describes the epidemiological, clinicpathological and ultrasonographic aspects of 28 cases of infection by Dioctophyma renale in dogs in the West Frontier region of Rio Grande do Sul, Brazil. Street dogs were the most affected and all of them had access to the banks of the Uruguay River. The renal and extra-renal lesions were predominantly characterized by atrophy of the renal parenchyma, sclerotic glomerulonephritis and granulomatous peritonitis, associated with free adult parasites and eggs in the abdominal cavity and erratic migration into the subcutaneous tissue. The ultrasound findings corresponded especially to the circular cross-sectional images with $0.6 \mathrm{~cm}$ in diameter at most, with hyperechoic margin and hypoechoic center too. These findings were pathognomonic for Dioctophyma renale infection and this examination was essential for the definitive diagnosis by clinical evaluation. The results of the study demonstrate the importance of this parasitism in the region, alert that the diagnosis has being underestimated, and point out the need to clarify its epidemiology in order to reach effective control measures.
\end{abstract}

INDEX TERMS: Dioctophymatosis, Dioctophyma renale, renal atrophy, ultrasonography, dogs.

RESUMO.- A infecção em cães por Dioctophyma renale, relatada em diversas partes do mundo, é considerada incomum, na maioria das vezes. No entanto, em algumas regiões são descritos números crescentes da infecção e muitos dados da epidemiologia e do ciclo biológico do parasito ainda são obscuros. Dessa forma, o trabalho tem como ob-

\footnotetext{
${ }^{1}$ Recebido em 19 de março de 2015.

Aceito para publicação em 14 de setembro de 2015.

${ }^{2}$ Programa de Pós-Graduação em Ciência Animal, área de concentração em Sanidade Animal/Patologia Veterinária, Universidade Federal do Pampa (Unipampa), Uruguaiana, RS 97500-970, Brasil.

${ }^{3}$ Médica Veterinária, Prefeitura Municipal de Uruguaiana, Uruguaiana, RS 97500-510.

${ }^{4}$ Setor de Clínica Cirúrgica de Pequenos Animais, Hospital Universitário Veterinário (HUVet), Unipampa, Uruguaiana, RS 97500-970.

${ }^{5}$ Setor de Radiologia Veterinária, HUVet-Unipampa, Uruguaiana, RS 97500-970.

${ }^{6}$ Laboratório de Patologia Veterinária (LPV), HUVet-Unipampa, Uruguaiana, RS 97500-970. *Autor para correspondência: anjosbl@gmail.com
}

jetivo descrever os aspectos epidemiológicos, clinicopatológicos e ultrassonográficos de casos de infecção por Dioctophyma renale em cães na região da Fronteira Oeste do Rio Grande do Sul. Foram estudados 28 casos de dioctofimose em cães necropsiados ou clinicamente avaliados, submetidos à ultrassonografia e cirurgia para retirada dos parasitos. Os cães errantes foram os mais acometidos e todos com possível acesso às margens do Rio Uruguai. As lesões renais e extrarrenais foram caracterizadas predominantemente por atrofia do parênquima renal com glomerulonefrite esclerosante e peritonite granulomatosa associada a parasitos adultos livres na cavidade abdominal e ovos, bem como migrações erráticas para o tecido subcutâneo. Por fim, os achados ultrassonográficos corresponderam, especialmente, a imagens transversais circulares de até $0,6 \mathrm{~cm}$ de diâmetro, com margem hiperecoica e centro hipoecoico. Esses achados foram patognomônicos para infecção por Dioctophyma renale, e o exame ultrassonográfico se mostrou indispensável para o diagnóstico definitivo durante a 
avaliação clínica. Os achados observados nesse estudo demonstram a importância dessa parasitose na região. Além disso, alertam para a importância do diagnóstico, que vem sendo subestimado, além de apontar a necessidade de mais dados acerca da epidemiologia da doença para que se chegue a métodos efetivos de controle.

TERMOS DE INDEXAÇÃO: Dioctofimatose, Dioctophyma renale, atrofia renal, ultrassonografia, caninos.

\section{INTRODUÇÃo}

Popularmente conhecido como verme gigante do rim, Dioctophyma renale é o maior nematódeo já descrito que parasita animais (Mech \& Tracy 2001) e também relatado em humanos (Sun et al. 1986, Katafigiotis et al. 2013). Os adultos têm morfologia cilíndrica, coloração vermelha e podem medir até um metro de comprimento (Monteiro et al. 2002, Leite et al. 2005, Alves et al. 2007). 0 parasito é encontrado frequentemente no rim direito, porém pode ser observado em rim esquerdo, cavidade abdominal, cavidade torácica, ureteres, bexiga e tecido subcutâneo de animais domésticos e silvestres (Kommers et al. 1999, Kano et al. 2003, Nakagawa et al. 2007, Milanelo et al. 2009, Verocai et al. 2009, Ishizaki et al. 2010, Zabott et al. 2012).

No Brasil, há relatos desse parasitismo em cães (Kommers et al. 1999, Monteiro et al. 2002, Kano et al. 2003, Leite et al. 2005, Pereira et al. 2006), gatos (Verocai et al. 2009) e de espécies silvestres como cachorro-do-mato Cerdocyon thous (Ribeiro et al. 2009), lobo-guará Chrysocyon brachyurus (Varzone et al. 2008), quati Nasua nasua (Milanelo et al. 2009), macaco Cebus apella (Ishizaki et al. 2010) e furão Galictis cuja (Zabott et al. 2012).

O ciclo biológico é complexo e, embora considerados de pouca importância para o ciclo, os cães são definidos como hospedeiros definitivos (HD) terminais ou anormais (Kommers et al. 1999, Pedrassani 2009). Os anelídeos aquáticos Lumbriculus variegatus são demonstrados como hospedeiros intermediários (HI), enquanto peixes de água doce e sapos Chaunus ictericus são considerados hospedeiros intermediários e paratênicos (HP) (Alves et al. 2007, Nakagawa et al. 2007, Pedrassani 2009).

Os nematódeos adultos se reproduzem comumente no rim direito do hospedeiro definitivo e os ovos são liberados na urina (Leite et al. 2005, Pedrassani 2009). A evolução ao estágio larval $\left(\mathrm{L}_{1}\right)$ pode variar, quanto mais alta a temperatura ambiental mais eficiente e rápido será o seu desenvolvimento (Pedrassani 2009). As $\mathrm{L}_{1}$, quando ingeridas pelo $\mathrm{HI}$, eclodem e passam a $\mathrm{L}_{2}$, quando formarão cistos no celoma do HI (Kano et al. 2003, Fortes 2004). 0 ciclo prossegue quando o HD ingere o HI ou HP com a larva $L_{3}$, e essa penetra na parede do estômago ou do duodeno, que pode migrar para a cavidade, onde frequentemente atinge o rim direito (Schimidt 1924, Pedrassani 2009).

Normalmente, a infecção em cães é subclínica e considerada incomum (Kommers et al. 1999, Kano et al. 2003). No entanto, alguns relatos no Rio Grande do Sul têm demonstrado que a ocorrência da infecção em cães é elevada e que a importância do cão no ciclo de transmissão e perpetuação da doença parecem subestimados (Colpo et al. 2007, Mistieri et al. 2014). Dessa forma, o presente traba- lho tem como objetivo descrever os aspectos epidemiológicos, clinicopatológicos e ultrassonográficos de casos de infecção por Dioctophyma renale em cães.

\section{MATERIAL E MÉTODOS}

Foi realizado levantamento de casos de dioctofimatose em cães atendidos no Hospital Universitário Veterinário (HUVet) da Universidade Federal do Pampa de 2011 a 2014. Na necropsia foram colhidos fragmentos de órgãos e tecidos, fixados em formol a 10\% e processados rotineiramente para avaliação histopatológica pela coloração de hematoxilina e eosina.

Em quatro cães a doença foi diagnosticada ainda na avaliação clínica de rotina e foram submetidos à avaliação ultrassonográfica e, quando cabível, à laparotomia exploratória, com remoção dos tecidos lesionados juntamente com os parasitos adultos, e nefrectomia unilateral. 0 acesso à região subcutânea inguinal e paraprepucial foi efetuado sem complicações, com rápida exposição e retirada dos parasitos. 0 acesso para laparotomia exploratória e nefrectomia foi realizado por incisão mediana pré-retroumbilical. Esses cães também foram submetidos à urinálise e o sedimento avaliado sob microscopia.

\section{RESULTADOS}

Foram avaliados por meio de necropsia e por avaliação clínica 28 cães parasitados por Dioctophyma renale, todos provenientes do município de Uruguaiana - RS. Desses cães, 24 foram recebidos para avaliação post mortem e quatro foram atendidos e avaliados na clínica e submetidos à cirurgia para retirada dos parasitos. 0 rim direito de um destes últimos foi encaminhado para avaliação histopatológica.

Dos 28 cães, 24 eram sem raça definida, dois Boxer, um Rottweiler e um Australian Cattle Dog. Dezessete machos, 11 fêmeas e 27 deles tinham idade superior a dois anos. Do total, 16 eram cães errantes, 12 eram criados em residência e todos tinham acesso a pátio gramado. Em 15 cães o parasito foi encontrado apenas no rim direito em meio a exsudado hemorrágico. Em cinco cães os exemplares de $D$. renale foram observados livres na cavidade abdominal e em outros cinco os vermes foram observados tanto no rim direito como livres no abdome. Em dois casos, o parasitismo foi encontrado no tecido subcutâneo na região inguinal e na região do flanco esquerdo. Em um caso foi observado, além do parasito livre na cavidade abdominal, um exemplar no interior de um cisto caudal ao rim direito. 0 número de parasitos variou entre um e 24 exemplares, machos e fêmeas.

Macroscopicamente, os cães apresentaram atrofia acentuada ou até mesmo ausência do parênquima renal do rim direito associado a exemplares de $D$. renale adultos. $\mathrm{Na}$ maioria dos casos, o rim encontrava-se aumentado de volume, com consistência flutuante, acentuada atrofia/perda do parênquima e cápsula fibrosa espessa preenchida por líquido serossanguinolento (aspecto cístico) em meio a um ou mais vermes (Fig.1). Em alguns casos observou-se acentuada atrofia renal por hidronefrose, caracterizada por achatamento da crista renal e compressão das regiões medular e cortical. Nos casos em que a atrofia era acentuada, o rim contralateral encontrava-se hipertrófico. Dos casos em que foram observados exemplares de $D$. renale livres na cavidade abdominal, havia peritonite acentuada caracterizada por exudato seroso, amarelado e com filamentos 

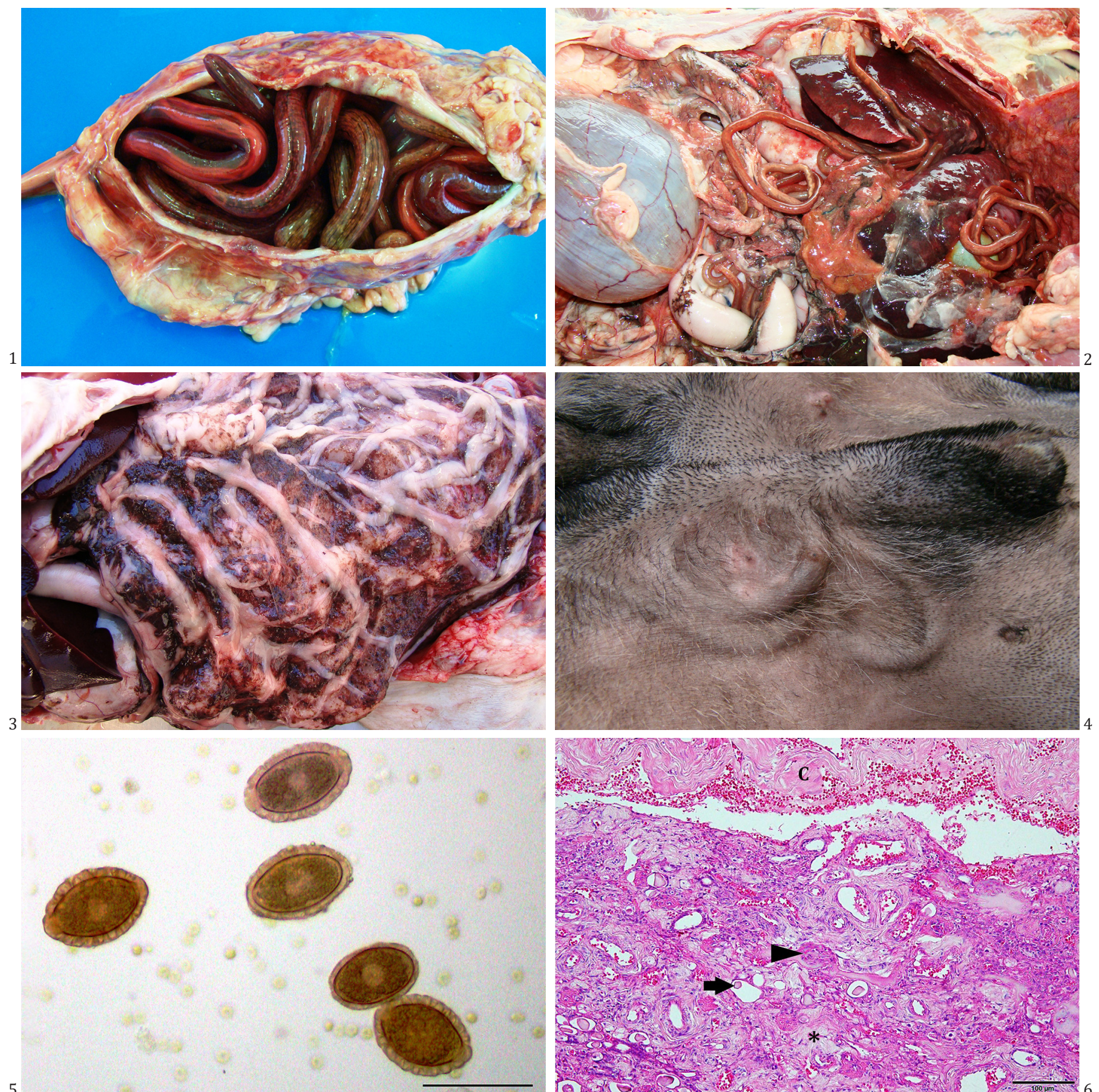

Fig.1. No rim direito de um cão observa-se espessamento da cápsula fibrosa e acentuada atrofia do parênquima renal (aspecto cístico) associados a exemplares de Dioctophyma renale.

Fig.3. Na cavidade abdominal de um cão com dioctofimose observa-se peritonite granulomatosa difusa acentuada com omento maior espessado e marrom-enegrecido. Notam-se pequenos nódulos em meio à gordura do omento.

Fig.5. Sedimento de urina de cão com ovos de nematoda com até $80 \mu \mathrm{m}$ de comprimento com morfologia compatível com Dioctophyma renale. Podem ser observados ovos de casca espessa com depressões na superfície e polos lisos, em meio a grande quantidade de hemácias. Barra $=100 \mu \mathrm{m}$.

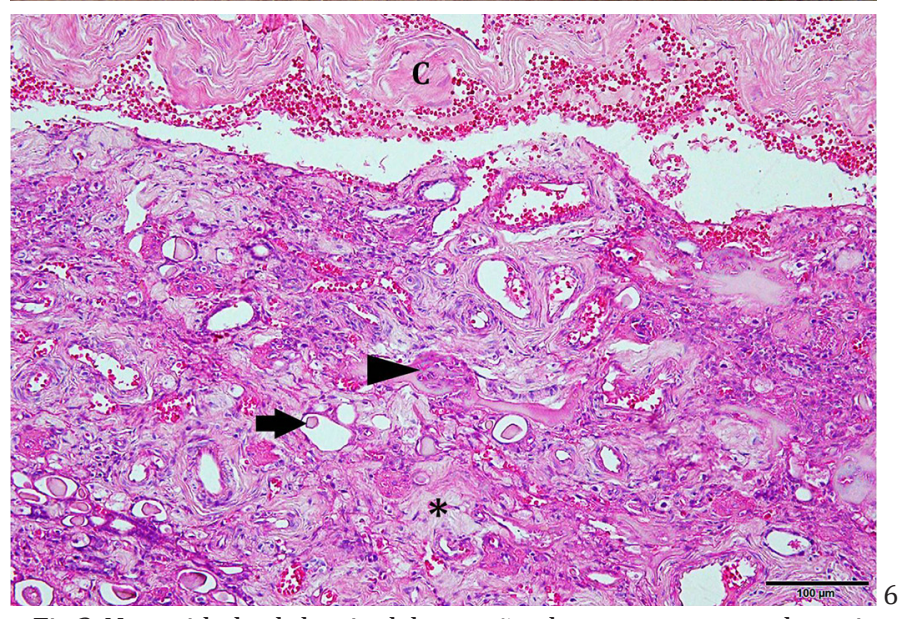

Fig.2. Na cavidade abdominal de um cão observa-se acentuada peritonite caracterizada por hiperemia da serosa, filamentos de fibrina aderidos na serosa dos órgãos associado a exemplares de Dioctophyma renale livres na cavidade. Nota-se a hiperemia do peritônio e a maior concentração dos parasitos próximo ao fígado.

Fig.4. Região paraprepucial de um cão com circunvoluções elevadas no subcutâneo, associadas à migração errática de exemplar de Dioctophyma renale.

Fig.6. No rim direito de um cão com dioctofimose observa-se glomerulonefrite esclerosante difusa acentuada com acentuada atrofia da região cortical e fibrose intensa. Notar a intensa diminuição dos glomérulos, muitos escleróticos (cabeça de seta) e túbulos renais, além do espessamento da capsula renal (C). Ainda há cilindros hialinos em túbulos renais remanescentes, alguns deles dilatados (seta). HE. Barra $=100 \mu \mathrm{m}$. 


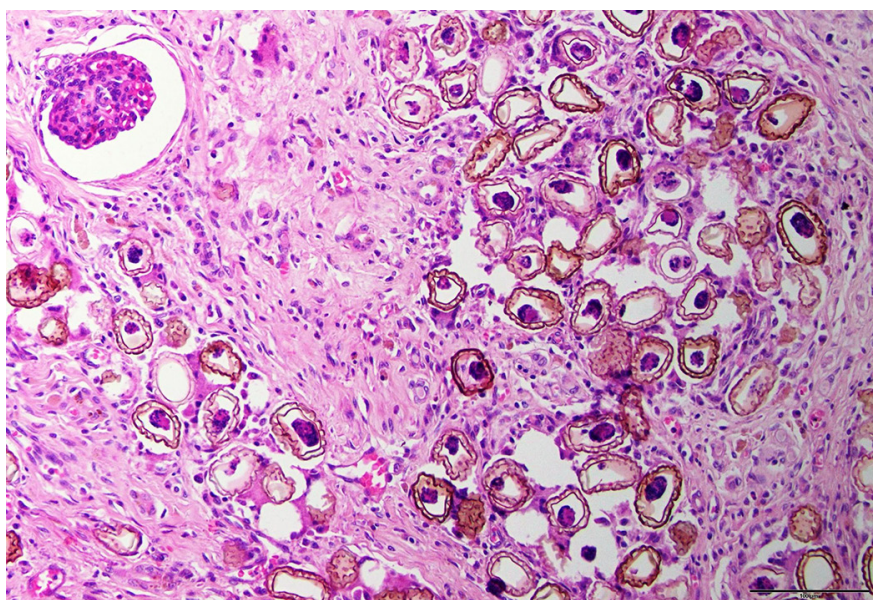

Fig.7. No rim direito de um cão observa-se glomerulonefrite granulomatosa com perda do parênquima renal e intensa fibrose associada a ovos de Dioctophyma renale com casca castanha e refringente. HE. Barra $=100 \mu \mathrm{m}$.

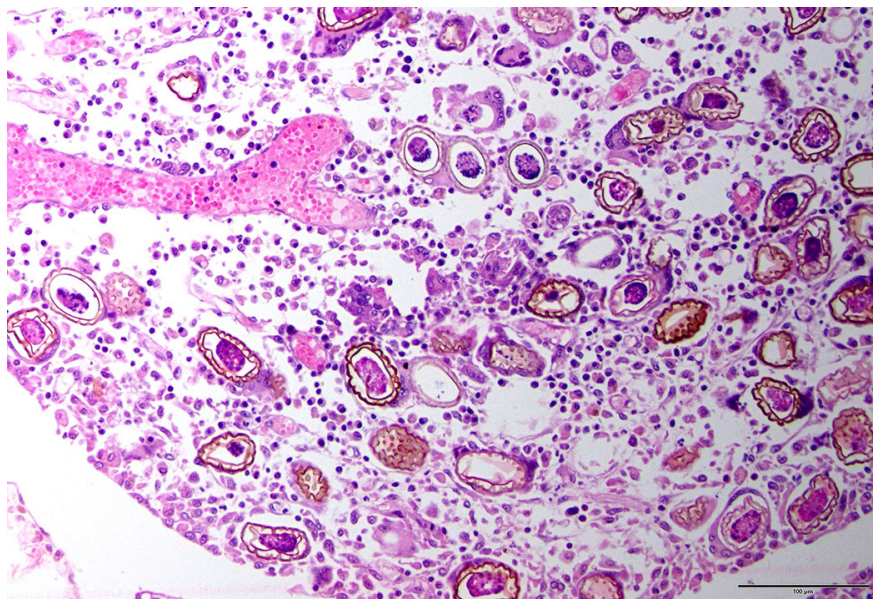

Fig.8. Intensa peritonite granulomatosa em um cão associada a inúmeros ovos fecundos de Dioctophyma renale. Podem ser observadas células gigantes multinucleadas com ovos fagocitados. Notar ovos com envoltório castanho e com depressões, exceto nos polos. HE. Barra $=100 \mu \mathrm{m}$.

de fibrina (Fig.2), em um cão e, em um segundo, o omento maior apresentava-se difusamente espessado e enegrecido (Fig.3). Nos cães em que o parasitismo foi observado no subcutâneo, notava-se, ao exame externo, aumento de volume irregular de aspecto cilíndrico e disposto de forma espiralada nas regiões do flanco e inguinal (Fig.4). Um dos cães apresentou ainda lesões erosivas na superfície capsular do fígado com $0,1 \mathrm{~cm}$ de diâmetro e trajetos subcapsulares com até $0,8 \mathrm{~cm}$ causadas por parasitos fêmeas jovens livres na cavidade. Foi coletada urina da bexiga de todos os cães necropsiados com parasitas fêmeas no rim direito, a qual apresentou grande quantidade de ovos fecundos de $D$. renale (Fig.5).

Histologicamente, na maior parte dos casos havia perda do parênquima renal com diminuição do número de glomérulos e túbulos renais. Observou-se acentuada esclerose glomerular com espessamento de cápsula de Bowman e intensa fibroplasia nas regiões cortical e medular. Os glomérulos escleróticos apresentavam-se diminuídos, hialini-

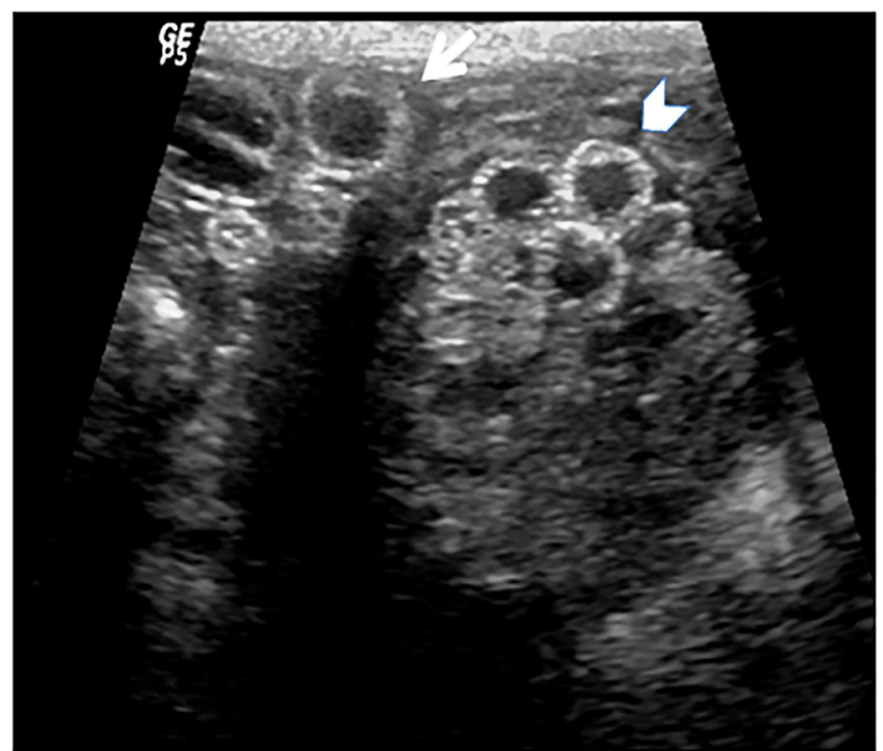

Fig.9. Ultrassonografia do abdome de cadela em plano transversal. Notam-se múltiplas estruturas com morfologia característica de Dioctophyma renale em rim direito (cabeça de seta). As estruturas são cilíndricas com parede hiperecoica e centro hipoecoico, envoltas por cápsula fibrosa. Observam-se estruturas semelhantes livres na cavidade abdominal (seta).

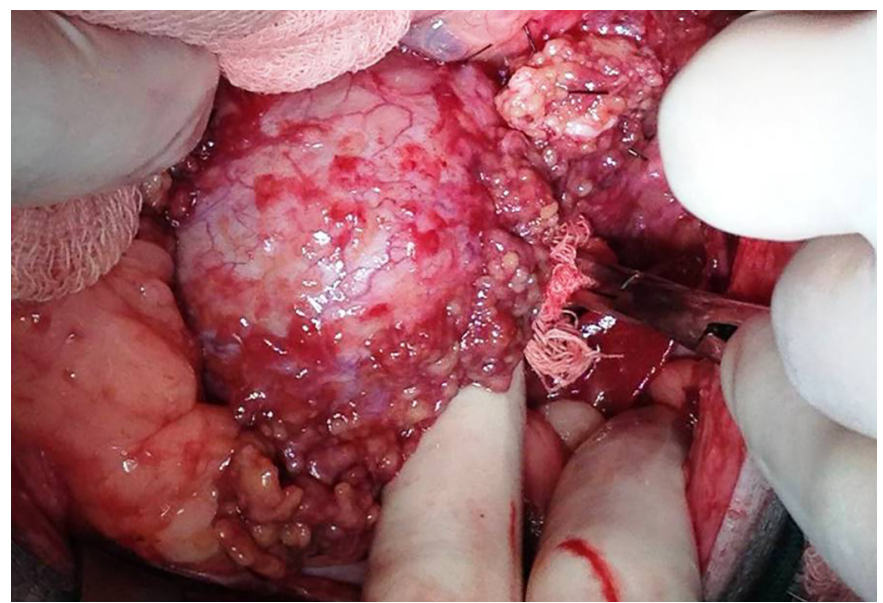

Fig.10. Imagem transoperatória de celiotomia exploratória de cão com dioctofimose renal e abdominal. Notar o rim direito após exposição cirúrgica com intensa neovascularização da cápsula renal e formações granulomatosas na gordura perirrenal.

zados com hipocelularidade e, por vezes, com perda total dos capilares do tufo glomerular. Foram observadas áreas com infiltrado inflamatório linfoplasmocitário no interstício e cilindros hialinos (Fig.6). Em alguns casos observou-se que o parênquima renal estava atrofiado, embora o epitélio da pelve estivesse preservado, apenas com compressão das camadas medular e cortical. Em um dos casos avaliados foi observada intensa deposição de ovos em meio ao parênquima renal com acentuada nefrite granulomatosa (Fig.7) e, em outro caso, havia no omento grande quantidade de células gigantes multinucleadas, algumas dessas fagocitando ovos de D. renale (Fig.8).

Em 2013 e 2014 foram diagnosticados quatro cães com D. renale durante exame clínico. Esses animais foram sub- 
metidos à avaliação clínica de rotina pré-cirúrgica ou por apresentarem emagrecimento progressivo e dor abdominal (dois cães). A infecção foi confirmada por avaliação ultrassonográfica. Os quatro cães foram submetidos a urinálise e ultrassonografia. A urinálise revelou grande quantidade de ovos de Dioctophyma renale em dois cães. Na avaliação ultrassonográfica foram observadas estruturas parasitárias em planos longitudinais e transversais compatíveis com $D$. renale. No plano longitudinal foram observadas estruturas alongadas com paredes hiperecoicas e, no plano transversal, foram observados halos hiperecoicos e conteúdo hipoecoico, com até $0,6 \mathrm{~cm}$ de diâmetro, tanto na cavidade abdominal como no rim direito (Fig.9) e no subcutâneo da região inguinal. Em três desses casos observaram-se estruturas parasitárias no rim direito em substituição ao parênquima e com preservação da cápsula renal, na região inguinal, livres na cavidade abdominal mesogástrica e epigástrica direita, bem como no tecido subcutâneo da região paraprepucial.

Os quatro cães foram submetidos a tratamento cirúrgico para remoção dos vermes livres no abdômen, na região subcutânea e no rim por nefrectomia, quando este estava acometido. Notou-se intensa neovascularização da cápsula renal, bem como aderência do rim direito à veia cava caudal e corpos vertebrais lombares em todos os casos em que houve parasitismo renal. Houve dificuldade para a exposição do hilo renal devida às intensas aderências. Outras alterações, como discreta ascite com deposição de material filamentar e congestão linfática foram observadas. Os parasitos livres na cavidade encontravam-se alojados predominantemente na região dorsolateral epigástrica direita, próximos ao lobo hepático direito. Em um desses animais foi observada peritonite granulomatosa acentuada (Fig.10) e áreas de hemorragia em segmentos do omento, gordura intra-abdominal e no mesentério.

\section{DISCUSSÃO}

O diagnóstico de dioctofimose pôde ser realizado com base nas características morfológicas do parasito e das lesões observadas nos diversos órgãos avaliados (Nakagawa et al. 2007, Ferreira et al. 2010). O exame ultrassonográfico se mostrou eficiente para o reconhecimento clínico dos casos. Dessa forma, foi possível minimizar os danos da infecção por auxiliar na condução dos casos à cirurgia (Zardo et al. 2012).

Nesse estudo, a presença de $D$. renale foi um achado incidental em aproximadamente $90 \%$ dos 28 cães avaliados. A maioria desses, encontrada na necropsia ou ao exame clínico sem terem apresentado previamente qualquer sinal clínico que indicasse o parasitismo, fato comumente observado por clínicos e patologistas na rotina de diagnóstico (Monteiro et al. 2002, Leite et al. 2005, Alves et al. 2007). Embora, na maioria dos casos, não sejam observados sinais clínicos, os cães podem apresentar apatia, anorexia, emagrecimento, marcha vacilante e hematúria (Alves et al. 2007). Ainda que incomum, ovos de $D$. renale na bexiga podem propiciar a agregação de urólitos a ponto de desencadear sinais clínicos característicos de doença obstrutiva do trato urinário inferior (Whelen et al. 2011).
Trabalhos que evidenciem o diagnóstico clínico e o tratamento do paciente são escassos e, em geral, apresentam relatos únicos (Ferreira et al. 2010). Os sinais clínicos variaram desde aumento de volume da região subcutânea, em decorrência de migração errática, até alterações generalizadas e pouco específicas, como emagrecimento progressivo, aborto e prostração (Lemos et al. 2010). Entretanto, por serem pouco específicas, acabam por dificultar a suspeita clínica da parasitose.

0 aparecimento de animais parasitados por Dioctophyma renale no Brasil pode estar intimamente relacionado ao potencial hídrico de algumas regiões do país, onde diversas cidades encontram-se localizadas à margem de rios. Essa característica favorece o acesso dos HD a áreas propícias ao desenvolvimento dos HI do nematódeo (Monteiro et al. 2002, Kano et al. 2003, Pereira et al. 2006). Nesse estudo os cães errantes foram os mais afetados. Esses vivem nas ruas e possuem acesso a áreas contaminadas e a grande diversidade de alimentos, como peixe cru, pois nesses locais as atividades de pesca e lazer são intensas (Monteiro et al. 2002, Kano et al. 2003). No município de Uruguaiana-RS, origem de todos os cães avaliados, o Rio Uruguai corre às margens das áreas norte e oeste da cidade, situação que pode favorecer a alta ocorrência da infecção nos cães. Nessas áreas, é comum a presença de pescadores que realizam a limpeza dos peixes às margens do rio e deixam as vísceras e sobras expostas. É importante salientar que mesmo os cães com proprietário podem ser cães semidomiciliados, com acesso irrestrito às ruas. Cães de fazenda podem estar igualmente expostos, embora nenhum caso proveniente de propriedades rurais da região da fronteira oeste tenha sido confirmado. Existem relatos feitos por médicos veterinários sobre a ocorrência dessa parasitose em outros municípios da fronteira oeste que são banhados pelo Rio Uruguai, o que reforça a hipótese de que o ciclo biológico do parasito está diretamente ligado ao acesso a essas áreas alagadas e ao desenvolvimento da infecção na espécie canina.

Embora pouco utilizado, o diagnóstico pode ser realizado através da identificação de ovos de Dioctophyma renale em exames parasitológicos de urina (Milanelo et al. 2009) e a urografia excretora (RX contrastado) (Kano et al. 2003). Mais comumente, pode se obter o diagnóstico através de avaliação ultrassonografia da região abdominal, o que auxilia na decisão pelo tratamento cirúrgico (Cottar et al. 2012, Zardo et al. 2012), e a presença dos parasitos adultos na ocasião de necropsia (Kommers et al. 1999, Alves et al. 2007). Em alguns casos o hemograma demonstra ocorrência de processo inflamatório (Ferreira et al. 2010), porém é inespecífico, na maioria dos casos. Em humanos também é utilizada a tomografia, porém, em algumas ocasiões, a suspeita de infecção por $D$. renale não é considerada e as imagens produzidas pela tomografia do rim ou da cavidade abdominal podem ser confundidas com massas tumorais (Sun et al. 1986).

A pesquisa de ovos do parasito na urina dos pacientes é empregada para estudos epidemiológicos da doença (Milanelo et al. 2009, Pedrassani 2009, Silveira et al. 2009). No presente levantamento, ovos de Dioctophyma renale foram observados em poucos casos, o que parece ser comum para 
essa parasitose (Mesquita et al. 2014). Essa avaliação acaba por subestimar os casos, uma vez que os ovos não são liberados constantemente e o cão pode não apresentar fêmeas adultas dos parasitos no rim.

As avaliações de imagem são imprescindíveis no diagnóstico definitivo quando há suspeita de dioctofimatose. 0 diagnóstico definitivo foi possível em todos os casos submetidos à ultrassonografia, independente da localização e do número de exemplares. As alterações e a visualização de D. renale são patognomônicas e incontestáveis (Soler et al. 2008, Cottar et al. 2012, Rahal et al. 2014). Foram observadas ainda alterações marcantes no omento, mesentério e peritônio, na laparotomia exploratória, não visualizadas na avaliação ultrassonográfica.

Em diversos estudos foi sugerido que a apresentação macroscópica da maioria dos casos de infecção por D. renale pode estar associada ao fato de que as larvas $\mathrm{L}_{3}$ penetram e atravessam na parede do estômago e permanecerem livres na cavidade abdominal (Kommers et al. 1999, Pereira et al. 2006, Pedrassani 2009), ou podem migrar para o fígado, onde se alimentam e se desenvolvem até L4, quando deixam o fígado e penetram no rim (geralmente o direito), e desenvolvem-se até o estádio adulto (Osborne et al 1969). Desta forma, o parasitismo no HD pode estar relacionado ao local de penetração das larvas na parede do trato digestório, o que explica a maior parte dos achados macroscópicos nas necropsias, ou acidentalmente, durante procedimentos cirúrgicos na cavidade abdominal.

A maioria dos cães com o parasitismo no rim apresentou acentuada destruição do parênquima, comum na dioctofimatose canina. Diversos autores relataram destruição progressiva das camadas cortical e medular, reduzindo o órgão a uma cápsula fibrosa (Kommers et al. 1999, Leite et al. 2005, Nakagawa et al. 2007, Ferreira et al. 2010). No entanto, grande parte da perda do parênquima renal deve ser associada também a hidronefrose (Nakagawa et al. 2007). Essa condição é causada pela obstrução do óstio ureteral interno pelos nematódeos adultos. A obstrução também determina atrofia com preservação do epitélio da pelve renal, conforme observado em alguns cães desse levantamento. Essa lesão deve ser considerada importante para o desenvolvimento do quadro clínico de insuficiência renal de alguns cães com dioctofimose (Osborne et al. 1969). Dois animais desenvolveram ainda peritonite granulomatosa difusa acentuada pela presença massiva de ovos embrionados, o que pode ser considerado um fator complicador do quadro clínico de cães infectados.

As lesões microscópicas do parênquima renal caracterizaram-se por perda do parênquima por atrofia, reação inflamatória intersticial mononuclear, glomeruloesclerose e fibrose. Esses achados são os mais comumente relatados em cães infectados (Kommers et al. 1999, Leite et al. 2005, Milanelo et al. 2009). Em um cão avaliado nesse estudo, foi observada intensa deposição de ovos em meio ao parênquima renal com acentuada nefrite granulomatosa, fato incomum para esse tipo de infecção.

Com base nos achados clinicopatológicos e ultrassonográficos relatados nesse estudo e a dificuldade de se estabelecer o diagnóstico clínico da doença, sugere-se que a ocorrência da infecção por Dioctophyma renale em cães na região é expressiva e vem sendo subestimada. Percebe-se que a inclusão de exames de imagem, como a avaliação ultrassonográfica abdominal, é determinante para o diagnóstico definitivo e precoce da doença. Tendo em vista ainda a alta ocorrência dessa parasitose na região da Fronteira Oeste do Rio Grande do Sul e a superficialidade das informações acerca da epidemiologia e biologia de Dioctophyma renale, mais estudos devem ser realizados para elucidar fatores determinantes para o desenvolvimento da doença em cães e seu real potencial zoonótico.

\section{REFERÊNCIAS}

Alves G.C., Silva D.T. \& Neves M.F. 2007. Dioctophyma renale: o parasita gigante do rim. Revta Cient. Eletrôn. Med. Vet. 8.

Colpo C.B., Silva A.S., Monteiro S.G., Stainki D.R., Camargo D.G. \& Colpo E.T.B. 2007. Ocorrência de Dioctophyma renale em cães no município de Uruguaiana. Revta FCVA, Uruguaiana, 14:175-180.

Cottar B.H., Dittrich G., Ferreira A.A., Carvalho A.C.P., Albernaz V.G.P., Luz M.T. \& Tasqueti U.I. 2012. Achados ultrassonográficos de cães parasitados por Dioctophyma renale: estudo retrospectivo. Vet. Zootec. 19(Supl.1):8-11.

Ferreira V.L., Medeiros F.P., July J.R. \& Raso T.F. 2010. Dioctophyma renale in a dog: clinical diagnosis and surgical treatment. Vet. Parasitol. 168(1/2):151-155.

Fortes E. 2004. Parasitologia Veterinária. 4aㅡ ed. Ícone Editora, São Paulo, p.370-371.

Ishizaki M.N., Imbelonib A.A., Munizb J.A.P.C., Scalerciob S.P.R.A., Benignoa R.R.M., Pereira W.L.A. \& Junior A.C.C.L. 2010. Dioctophyma renale (Goeze, 1782) in the abdominal cavity of a capuchin monkey (Cebus apella), Brazil. Vet. Parasitol. 173(3/4):340-343.

Kano F.S., Shimada M.T., Suzuki S.N., Osaki S.C., Menarim B.C., Ruthes F.R.V. \& Filho M.A.L. 2003. Ocorrência da dioctofimose em dois cães no município de Guarapuava/PR. Semina, Ciênc. Agrárias 24(1):177-180.

Katafigiotis I., Fragkiadis E., Pournaras C., Nonni A. \& Stravodimos K.G. 2013. A rare case of a 39 years old male with a parasite called Dioctophyma renale mimicking renal cancer at the computed tomography of the right kidney: a case report. Parasitol. Int. 62:459-460.

Kommers G.D., Ilha M.R.S. \& Barros C.S.L. 1999. Dioctofimose em cães: 16 casos. Ciência Rural 29(3):517-522.

Leite L.C., Cirio S.M., Diniz J.M.F., Luz E., Navarro-Silva M.A., Silva A.W.C., Leite S.C., Zadorosnei A.C., Musiat K.C., Veronesi E.M. \& Pereira C.C. 2005. Lesões anatomopatológicas presentes na infecção por Dioctophyma renale (Goze, 1782) em cães domésticos (Canis familiaris Linnaeus, 1758). Archs Vet. Sci. 10(1):95-101.

Lemos L.S., Santos A.S.O., Rodrigues A.B.F., Goulart M.L.V.S., Almeida L.G. \& Silveira L.S. 2010. Extrarenal lesion caused by Dioctophyma renale eggs in an erratic cycle in a dog. Int. J. Morphol. 28(4):1031-1034.

Mech L.D. \& Tracy S.T. 2001. Prevalence of gigant kidney worm (Dioctophyma renale) in wild Mink (Mustela vison) in Minnesota. American Midland Naturalist 145(1):206-209.

Mesquita L.R., Rahal S.C., Faria L.G., Takahira R.K., Rocha N.S., Mamprim M.J. \& Oliveira H.S. 2014. Pre- and post operative evaluations of eight dogs following right nephrectomy due to Dioctophyma renale. Vet. Quart. 34(3):167-171.

Milanelo L., Moreira M.B., Fitorra L.S., Petri B.S.S., Alves M. \& Santos A.C. 2009. Occurrence of parasitism by Dioctophyma renale in ring-tailed coatis (Nasua nasua) of the Tiete Ecological Park, São Paulo, Brazil. Pesq. Vet. Bras. 29(12):959-962.

Mistieri M.L.A., Pascon J.P.E. \& Santos F.P. 2014. Infestação múltipla de Dioctophyme renale em cães portadores de leishmaniose em Uruguaiana, RS: relato de cinco casos. Revta Bras. Med. Vet. 36(2):195-198.

Monteiro S.G., Sallis L.S.V. \& Stainki D.R. 2002. Infecção natural por trinta e quatro helmintos da espécie Dioctophyma renale (Goeze, 1782) em um cão. Revta Fac. Zootec. Vet. Agron. Uruguaiana, 9(1):95-99. 
Nakagawa T.L.D.R., Bracarense A.P.F.R.L., Reis A.C.F., Yamamura M.H. \& Headley S.A. 2007. Giant kidney worm (Dioctophyma renale) infections in dogs from Northern Paraná, Brazil. Vet. Parasitol. 145:366-370.

Osborne C.A., Stevenes J.B., Hanlon G.F., Rosin E. \& Bemrick W.J. 1969. Dioctophyma renale in a dog. J. Am. Vet. Med. Assoc. 155(4):605-620.

Pedrassani D. 2009. Aspectos morfológicos, imunológicos e epidemiológicos de Dioctophyme renale em cães no distrito de São Cristóvão, Três Barras, Santa Catarina. Tese de Doutorado em Medicina Veterinária Preventiva, Faculdade de Ciências Agrárias e Veterinárias, Universidade Estadual Paulista, Jaboticabal, SP. 118p.

Pereira B.J., Girardelli G.L., Trivilin L.O., Lima V.R., Nunes L.C. \& Martins I.V.F. 2006. Ocorrência de dioctofimose em cães necropsiados do município de Cachoeiro do Itapemirim, Espírito Santo, Brasil, no período de maio a setembro de 2004. Revta Bras. Parasitol. Vet. 15(3):123-125.

Rahal S.C., Mamprim M.J., Oliveira H.S., Mesquita L.R., Faria L.G., Takahira R.K., Matsubara L.M. \& Agostinho F.S. 2014. Ultrasonographic, computed tomographic, and operative findings in dogs infested with giant kidney worms (Dioctophyme renale). J. Am. Vet. Med. Assoc. 244(5):555-558.

Ribeiro C.T., Verocai G.G. \& Tavares L.E.R. 2009. Dioctophyme renale (Nematoda, Dioctophymatidae) infection in the Crab-eating Fox (Cerdocyon thous) from Brazil. J. Wildl. Dis. 45(1):248-250.

Schimidt F. 1924. Contribuição para uma patologia veterinária no Rio Grande do Sul. Egatea 9:245-254.
Silveira L.L., Lemos L.S., Ferreira F.S., Freitas M.V., Pereira M.A.C. \& Carvalho C.B. 2009. Avaliação entre as técnicas de centrífugo-sedimentação e sedimentação rápida (Paratest ${ }^{\circledR}$ ) na identificação de ovos de Dioctophyma renale em urina de cães. J. Bras. Ciênc. Anim. 2(3):150-158.

Soler M., Cardoso L., Teixeira M. \& Agut A. 2008. Imaging diagnosis: Dioctophyma renale in a dog. Vet. Radiol. Ultrasound 49(3):307-308.

Sun T., Turnbull A., Lieberman P.H. \& Sternberg S.S. 1986. Giant kidney worm (Dioctophyma renale) infection mimicking retroperitoneal neoplasm. Am. J. Surg. Pathol. 10:508-512.

Varzone J.R.M., Aquino L.P.C.T. \& Rodovalho M.V.T. 2008. Achados macroscópicos de lesões resultantes do parasitismo por Dioctophyma renale em lobo-guará (Chrysocyon brachyurus): relato de caso. Ensaios e Ciência, Ciências Biológicas, Agrárias e da Saúde 12(2):171-178.

Verocai G.G., Measures L.N., Azevedo F.D., Correia T.R., Fernandes J.I. \& Scott F.B. 2009. Dioctophyma renale (Goeze, 1782) in the abdominal cavity of a domestic cat from Brazil. Vet. Parasitol. 161:342-344.

Whelen J.C., Houston D.M., White C. \& Favrin M.G. 2011. Ova of Dioctophyme renale in a canine struvite urolith. Can. Vet. J. 52(12):1353-1355.

Zabott M.V., Pinto S.B., Viott A.M., Tostes R.A., Bittencourt L.H.F.B., Konell A.L. \& Gruchouskei L. 2012. Ocorrência de Dioctophyma renale em Galictis cuja. Pesq. Vet. Bras. 32(8):786-788.

Zardo K.M., Santos D.R., Babicsak V.R., Belotta A.F., Oliveira H.S., Estanislau C.A., Mamprim M.J. \& Brandão C.V.S. 2012. Aspecto ultrassonográfico da dioctofimose renal canina. Vet. Zootec. 19(Supl.1):57-60. 